\title{
Nitrigreen a Borracha Ecológica
}

\author{
Alex S. Sirqueira, Raquel Reis, Lys Sirelli, Márcia V. 0. Silva, Ronaldo V. Monteiro \\ Nitriflex S/A Industria e Comércio
}

Resumo: Uma nova linha de borracha nitrílica pré-plastificada em óleo vegetal (Nitrigreen) com médio-alto teor de acrilonitrila (33\%) foi especialmente desenvolvida pela empresa Nitriflex S/A Ind. Com., para suprir as necessidades do mercado de borracha. A Nitrigreen é um produto que está de acordo com a diretiva européia 2005/84/EC, pois utiliza um plastificante de fonte renovável. Esta borracha tem efeito superior na redução da viscosidade Mooney do composto formulado quando comprado com as borrachas pré-plastificadas com dioctil ftalato (DOP). Além da facilidade de processamento, a Nitrigreen tem compatibilidade com os aditivos de vulcanização, podendo ser utilizada em formulações de artefatos de baixa dureza, como brinquedos, cilindros gráficos e artigos técnicos. A nova borracha nitrílica pré-plastificada com 50 partes de plastificante vegetal reduz sensivelmente o tempo de vulcanização dos compostos, tem excelente processabilidade, baixo consumo de energia, facilidade na incorporação de aditivos, principalmente cargas reforçadoras como o negro de fumo, além de manter as propriedades mecânicas semelhantes à da borracha pré-plastificada com DOP.

Palavras-chave: Borracha nitrílica, plastificantes, óleo vegetal, vulcanização, DOP.

\section{Nitrigreen the Ecological Rubber}

Abstract: New vegetable oil pre-plasticized rubbers (Nitrigreen) with 33\% of acrilonitrile were especially developed by Nitriflex to supply the rubber market necessities. Nitrigreen complies with European regulation 2005/84/EC as it uses a plasticizer from renewable source. This rubber has superior effect in the Mooney viscosity reduction compared with usual plasticizer such as phthalates. In addition to the ease of processing, Nitigreen is compatible with vulcanization additives and may be used in low hardness products, including toys, cylinders and technical parts. This new pre-plasticized nitrile rubber with $50 \mathrm{phr}$ of plasticizer has excellent processability, low consumption of energy, and facilitates incorporation of additives, especially reinforcing loadings such as carbon black. The mechanical properties of this rubber are slightly superior to those of a rubber containing DOP.

Keywords: Nitrile rubber, plasticizer, vegetable oil, vulcanization, DOP.

\section{Introdução}

O uso de aditivos na indústria de polímeros é recorrente da necessidade de aprimorar as propriedades/características finais dos artefatos/peças tais como, dureza, brilho, textura, resistência mecânica, etc. ${ }^{[1]}$. Entretanto a maioria dos aditivos são sintéticos e não biodegradáveis, tornando o artefato pós-uso um veículo de contaminação dos solos, rios e do ar. Em alguns casos, como dos plastificantes, pode ocorrer a exsudação do aditivo para a superfície da peça, ficando exposta a ação da temperatura, oxidação, radiação UV, etc. o que aumenta a contaminação por agentes químicos dos depósitos sanitários (lixões) e aterros das grandes cidades ${ }^{[2-6]}$.

O processamento da borracha sem a presença de plastificantes seria uma alternativa radical para eliminar esse problema, entretanto não seria de uso prático, em virtude do alto consumo de energia e tempo de processamento. Pensando numa alternativa que fosse capaz de conciliar a facilidade de processamento e ao mesmo tempo reduzir o impacto ambiental, a Nitriflex S/A Ind. Com. desenvolveu uma nova linha de borracha pré-plastificada (estendida em plastificantes) em óleo vegetal. Por ser um plastificante de fonte renovável, não poluente e biodegradável o óleo vegetal atendeu aos requisitos básicos para iniciar o desenvolvimento. A Nitrigreen tem a mesma facilidade de processamento de uma borracha pré-plastificada com outros plastificantes sintéticos convencionais tais como os ftalatos. A Nitrigreen é um produto que atende a diretiva européia 2005/84/EC ${ }^{[7]}$ que restringe o uso do DOP (dioctil ftalato) para artefatos infantis, em função da exsudação do DOP. Existe uma ampla discussão no meio científico sobre as causas da contaminação pelo DOP, principalmente em crianças.

Neste estudo será apresentado um comparativo entre a Nitrigreen e uma borracha pré-plastificada com DOP ${ }^{[6,8,9]}$.

\section{Experimental}

\section{Materiais}

A Tabela 1 apresenta as principais características das borrachas pré-plastificadas em óleo vegetal modificado (produto sob sigilo de patente) e com dioctil ftalato, ambas produzidas pela Nitriflex S/A Ind. Com.

Autor para correspondência: Alex S. Sirqueira, Nitriflex S/A Industria e Comércio, Rua Marumbi 1300, Campos Elíseos, CEP: 25221-000, 
Tabela 1. Características das borrachas.

\begin{tabular}{lcc}
\hline \multicolumn{1}{c}{ Propriedades } & DPFlex & Nitrigreen \\
\hline Teor de acrilonitrila ${ }^{\mathrm{a}}(\%)$ & 33 & 33 \\
Teor de plastificante $\left(\mathrm{phr}^{\mathrm{b}}\right)$ & $50(\mathrm{DOP})$ & 50 (Óleo vegetal) \\
Solubilidade em MEC $(\%)$ & 98 & 98 \\
Densidade $\left(\mathrm{g} / \mathrm{cm}^{3}\right)$ & 1,04 & 1,04 \\
Viscosidade mooney & 45 & 45 \\
$\left(\mathrm{MML}\left(1+4\right.\right.$ @ $\left.\left.100{ }^{\circ} \mathrm{C}\right){ }^{\mathrm{c}}\right)$ & & 33 \\
$\begin{array}{l}\text { Extração a quente em soxh- } \\
\text { let com eter etílico }(\%)\end{array}$ & 33 & \\
\hline aPolímero base; ${ }^{b}$ parte por cem de polímero; e ${ }^{\mathrm{c}}$ viscosidade moo- \\
ney da borracha laminada.
\end{tabular}

A formulação apresentada na Tabela 2 é específica para compostos de baixa dureza como cilindros de impressão. Os aditivos de vulcanização foram obtidos em fornecedores locais.

\section{Métodos}

As misturas foram processadas em misturador de rolos, marca Luxor, nas seguintes condições: temperatura $50{ }^{\circ} \mathrm{C}$, razão de fricção entre os cilindros de 1:1,25, tempo total de mistura de 15 minutos. Mediu-se a variação da massa após o processamento bem como a temperatura do composto, considerando $2 \%$ como variação aceitável na massa do composto.

As borrachas DPFlex e Nitrigreen não vulcanizadas foram extraídas em éter etílico com auxílio de aparelho soxhlet por 24 horas.

Os parâmetros de vulcanização foram determinados através do reômetro de disco oscilatório, marca Monsanto $100 \mathrm{~S}$ a $160{ }^{\circ} \mathrm{C}$ com arco de oscilação de $1^{\circ}$. As propriedades físico-mecânicas analisadas foram: resistência à tração, rasgamento, dureza, resiliência, deformação permanente à compressão, resistência ao envelhecimento térmico e ensaio de inchamento em solventes. Para as avaliações físico-mecânicas considerou-se $2 \%$ de variação nos valores.

A variação de propriedades após o ensaio de envelhecimento e imersão em solvente foi determinado utilizando a Equação 1 a seguir:
Variação de propriedade $=$

(valor após o ensaio de envelhecimento valor antes do envelhecimento)/

valor antes do envelhecimento

A análise de calorimetria exploratória diferencial (DSC) foi utilizada na determinação da temperatura de transição vítrea ( $\mathrm{Tg}$ ) das borrachas não vulcanizadas, nas seguintes condições: faixa de temperatura de $-100{ }^{\circ} \mathrm{C} \mathrm{a}+20{ }^{\circ} \mathrm{C}$, com taxa de aquecimento de $10^{\circ} \mathrm{C} / \mathrm{min}$.

\section{Resultados e Discussão}

A Figura 1 apresenta os resultados da temperatura de transição vítrea das borrachas puras não vulcanizadas. Nota-se que a Nitrigreen (borracha pré-plastificada com óleo vegetal) tem o mesmo valor da temperatura de transição vítrea (Tg) da borracha pré-plastificada com dioctil ftalato, DPFlex. Por esse resultado nota-se que o óleo vegetal modificado atua de maneira semelhante ao DOP na redução da Tg da borracha nitrílica.

A Figura 2 ilustra a curva de vulcanização obtida através do reômetro de disco oscilatório a temperatura de $160{ }^{\circ} \mathrm{C}$, dos compostos formulados com a Nitrigreen e a DPFlex. Nota-se que para ambos os compostos o valor de torque mínimo foi o mesmo, entretanto o valor do tempo de segurança para a borracha com DOP foi maior. Os valores de torque máximo foram superiores para a DPFlex, esse resultado sugere que a

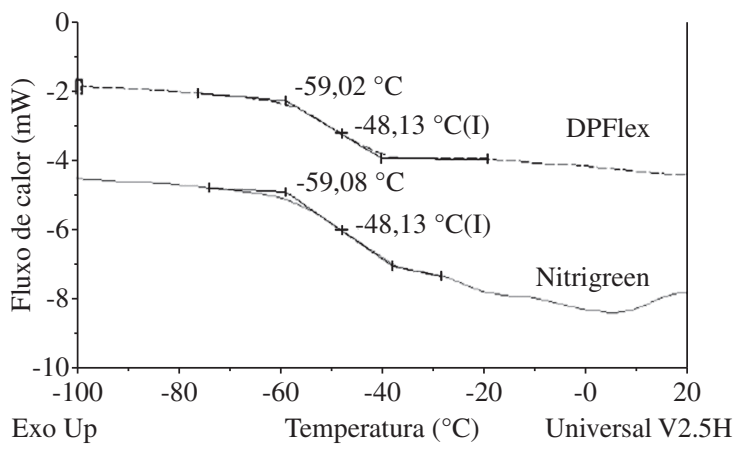

Figura 1. Curvas DSC das borrachas puras não vulcanizadas.

Tabela 2. Formulação para compostos de baixa dureza.

\begin{tabular}{lcrcc}
\hline \multicolumn{1}{c}{ Matéria-prima } & Função & \multicolumn{2}{c}{ Formulação (phr) } & Fornecedores \\
\cline { 3 - 4 } & & F1 & F2 & Nitriflex \\
Nitrigreen & Borracha & 150,0 & - & Nitriflex \\
DPFlex & Borracha & - & 150,0 & Brasóxido \\
Óxido de zinco & Ativador & 3,0 & 3,0 & \\
Ácido esteárico & Ativador & 0,5 & 0,5 & Cabot \\
Negro de fumo N990 & Carga & 65,0 & 65,0 & Struktol \\
Octamine & Antioxidante & 2,5 & 2,5 & Flexsys \\
TMTD & Acelerador & 1,0 & 1,0 & Flexsys \\
Enxofre & Agente de reticulação & 0,3 & 0,3 & Flexsys \\
MBTS & Acelerador & 1,5 & 1,5 & Struktol \\
Struktol WB -212 & Auxiliar de processo & 2,0 & 2,0 & 225,8 \\
Total & & 22,8 & & \\
\hline
\end{tabular}




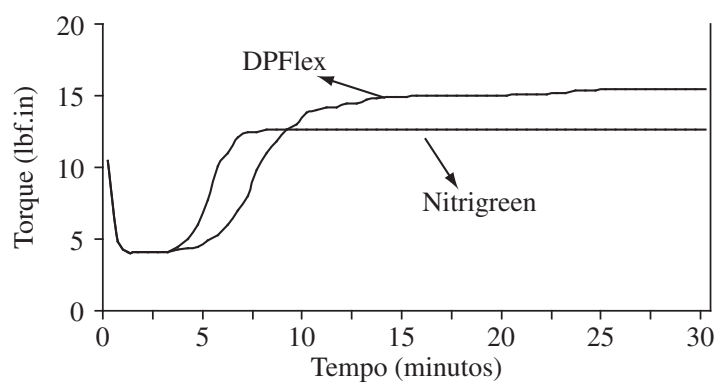

Figura 2. Curvas de torque x tempo obtidas em reômetro de disco oscilatório para borrachas.

Nitrigreen tem menor módulo elástico e menor dureza, comparado com a DPFlex. Esse resultado é interessante pois os artefatos produzidos com borrachas pré-plastificadas são de baixa dureza. Outro dado importante na reometria de disco oscilatório é o tempo de cura (t90). Nota-se pela Figura 2, considerável redução no tempo de vulcanização da Nitrigreen, a energia gasta para produzir o vulcanizado é menor ${ }^{[10]}$.

A Tabela 3 apresenta os resultados das propriedades físico-mecânicas antes e após os ensaios de inchamento em solventes e envelhecimento em estufa com circulação forçada de ar a $100{ }^{\circ} \mathrm{C}$ por 72 horas. De maneira geral as propriedades mecânicas da Nitrigreen foram superiores a da DPFlex, exceto para deformação permanente a compressão onde a DPFlex teve melhor performance. Vale destacar a redução de cinco pontos na dureza do composto com a borracha Nitrigreen, esse comportamento é bastante interessante pois a formulação para cilindros de baixa dureza. Pode-se atribuir esse comportamento a melhor interação do óleo vegetal com a borracha, uma vez que não foi observada exsudação após o processamento. Atribui-se os resultados encontrados a melhor dispersão do óleo vegetal entre as cadeias da borracha, o que ocasiona menor dureza e maior deformação permanente, pois houve uma maior plastificação do composto.

Não houve variação significativa na dureza dos compostos Nitrigreen e DPFlex após o ensaio em estufa com circulação forçada de ar, sugerindo o mesmo nível de degradação em ambas as composições.

Para os ensaios de inchamento com óleos e solventes nota-se que a Nitrigreen teve desempenho superior a DPFlex, como mostrado pela baixa variação no volume após imersão. Esses resultados corroboram os valores das propriedades mecânicas. A Nitrigreen está mais plastificada (maior afinidade do óleo com a borracha) o inchamento/extração será menor. Para verificar se há algum tipo de interação química entre a borracha e o óleo vegetal foram realizados testes de extração em soxhlet da borracha pura, os valores são apresentados na Tabela 1. Nota-se que não há nenhum tipo de interação química entre o óleo vegetal e a borracha nitrílica, logo o aumento na resistência ao inchamento pode ser atribuído a boa interação física entre o óleo vegetal e a borracha.

\section{Conclusões}

Pelos resultados obtidos pode-se concluir que a substituição do DOP pelo óleo vegetal é viável.

Houve redução significativa no tempo de vulcanização para Nitrigreen, logo a energia necessária para produzir o vulcanizado foi menor.

Tabela 3. Propriedades físico-mecânicas dos compostos vulcanizados.

\begin{tabular}{|c|c|c|}
\hline Resultados & F1 - Nitrigreen & F2 - DPFlex \\
\hline Resistência ao rasgamento (KN/m) & 23,0 & 22,6 \\
\hline Tensão de ruptura (MPa) & 9,2 & 7,0 \\
\hline Alongamento na ruptura (\%) & 709 & 569 \\
\hline Módulo a 300\% (MPa) & 2,8 & 3,7 \\
\hline Deformação permanente (\%) & 20,4 & 17,5 \\
\hline Dureza (Shore A) & 34 & 39 \\
\hline Resiliência (\%) & 55 & 55 \\
\hline Peso específico $\left(\mathrm{g} / \mathrm{cm}^{3}\right)$ & 1,138 & 1,138 \\
\hline \multicolumn{3}{|c|}{ Envelhecimento em estufa c/ circ. de ar (Temperatura $100{ }^{\circ} \mathrm{C}$, tempo 70 horas) } \\
\hline Variação de dureza (Shore A) & 5,00 & 6,00 \\
\hline \multicolumn{3}{|c|}{ Imersão em óleo IRM 903 (Temperatura $100{ }^{\circ} \mathrm{C}$, tempo 70 horas) } \\
\hline Variação de dureza (Shore A) & 9,00 & 8,00 \\
\hline Variação no volume (\%) & $-8,90$ & $-12,10$ \\
\hline \multicolumn{3}{|c|}{ Imersão em FUEL C (Temperatura $100{ }^{\circ} \mathrm{C}$, tempo 70 horas) } \\
\hline Variação de dureza (Shore A) & 0,00 & 0,00 \\
\hline Variação de volume (\%) & 1,00 & 2,10 \\
\hline \multicolumn{3}{|c|}{ Imersão em solvente de limpeza de cilindro (Temperatura $100{ }^{\circ} \mathrm{C}$, tempo 70 horas) } \\
\hline \multicolumn{3}{|l|}{ Solvente para limpeza de cilindros } \\
\hline Variação de dureza (Shore A) & 1,00 & 1,00 \\
\hline Variação de volume (\%) & 0,70 & 2,04 \\
\hline
\end{tabular}


As propriedades físico-mecânicas dos compostos com a Nitrigreen foram ligeiramente superiores a dos compostos com DOP.

A dureza do composto com a Nitrigreen foi menor favorecendo seu uso em artefatos macios.

A Nitrigreen é uma alternativa para produção de artefatos de baixa dureza menos poluente.

\section{Referências Bibliográficas}

1. Rabello, M. - “Aditivação de Polímeros”. Artliber, São Paulo, 2000.

2. Djilani, S. E.; Bouchami, T.; Krid, F.; Boudiaf, N. \& Messadi, D. - Eur.Polymer Journal, 36, p.1981 (2000).

3. Jiménez, A.; Torres, L. \& Kenny, J. M. - Polymer Degradation and Stability, 73, p.447 (2001).
4. Rahman, M. \& Brazel, C. S. - Progress in Polymer Science, 29, p.1223 (2004).

5. Acessado em 23/12/2007 www.cdc.gov.nceh/dls/report/

6. Marcilla, A.; Garcia, S. \& Quesada, J. C. G. - Polymer Testing, 27, p.221 (2008).

7. Directive 2005/84/EC of the European Parliament and of the Council.

8. Zanin, M. - "Resíduos plásticos e reciclagem - Aspestos gerais e tecnologia", EdUFSCar (2004).

9. Patino, J. M. R.; Sánchez, C. C. \& Nino, M. R. - Adv. Coll. Inter, Sci., 114, p.1 (2005).

10. Menon, A. R. R.; Pillai, C. K. S. \& Nando, G. B. - Eur. Polymer Journal, 34, p.923 (1998).

Enviado: $18 / 06 / 08$

Reenviado: 14/07/08

Aceito: 18/07/08 